\title{
A General Approach for Evaluating the Outage Probability in Microcellular Mobile Radio Systems*
}

\author{
A. Annamalai ${ }^{1}$ and C. Tellambura ${ }^{2}$ \\ 1. Department of Electrical and Computer Engineering, University of Victoria, PO Box 3055 STN CSC, Victoria \\ BC V8W 3P6, Canada, Tel: +1-250-721-6043, Fax: +1-250-721-6048, E-mail: annamalai@ece.uvic.ca \\ 2. School of Computer Science and Software Engineering, Monash University, Clayton, Victoria 3168, Australia \\ Tel: +1-613-9905-3296, Fax: +1-613-9905-3402,E-mail: chintha@dgs.monash.edu.au
}

\begin{abstract}
A unified expression for computing the probability of outage in cellular mobile radio systems is derived. The method handles non-integer Nakagami fading indexes, unequal Rice factors, dissimilar shadowing spreads, unequal transmit powers as well as all the common fading distributions (Rayleigh, Rice, Nakagami-m, Nakagami-q, lognormal-Rice, Suzuki and lognormal-Nakagami-m). The exact outage probability is expressed in terms of a finite-range integral. The integral can also be approximated by a Gauss-Chebychev quadrature (GCQ) formula requiring the knowledge of the moment generating function (MGF) at only a small number of points. An estimate of the remainder term is also derived. This technique lends itself to a powerful tool for outage analysis since it does not impose any restrictions while being easy to program. Some previous studies have suggested approximating Rician desired signal statistics by a Nakagami-m model to circumvent the difficulty in evaluating the outage in Rician fading. We asses the suitability of this approximation by providing a comparison study of the outage performance in these two fading conditions. Surprisingly, some basic results for Nakagami-m channel have been overlooked, which has led to misleadingly optimistic results with the Nakagami-m approximation model.
\end{abstract}

\section{INTRODUCTION}

In cellular radio systems, the spectrum utilization efficiency may be improved by reducing the cluster size but at the expense of increased cochannel interference $(\mathrm{CCl})$. The probability of outage (hereafter, simply referred to as outage) is a useful statistical measure of performance in the presence of CCI [1]. The outage performance of digital radio systems has been studied extensively in literature (see [1-8] among many others).

Consider evaluating the probability of outage in an interference-limited mobile fading environment. The instantaneous signal powers are modelled as random variables (RVs) $p_{k}$, $k=0,1,2, \ldots, L$, with mean $\bar{p}_{k}$. The subscript $k=0$ denotes the desired user signal, and $k=1, \ldots, L$ are for the interfering signals. The outage is given by,

$$
P_{\text {out }}=\operatorname{Pr}\left\{q I>p_{0}\right\}
$$

where $I=p_{1}+\ldots+p_{L}$ and $q$ is the power protection ratio, which is fixed by the type of modulation and transmission technique employed and the quality of service desired. Typically, $9<q<20$ (dB). For instance, $q=9.5 d B$ for the digital pan-European GSM system using GMSK modulation.

* This work was supported in part by a Strategic Project Grant from the Natural Sciences and Engineering Research Council (NSERC) of Canada and in part by BC Tel Mobility Cellular.
Numerous ad-hoc attempts have been made 10 obtain closed-form expressions for the outage under different fading scenarios. To get explicit formulas, it is often necessary to make restrictive assumptions (e.g., Nakagami fading severity parameter be a positive integer [2] or identical statistical distributions for all the interferers [7]) or approximations (replacing; a Rician RV by a Nakagami RV). Although the assumption that all the received signals (both desired and undesired) have the same statistical characteristics is quite reasonable for medium and large cell systems, its validity for microcellular systems is questionable. This is because an undesired signal from a distart cochannel cell may well be modelled by Rayleigh statstics but Rayleigh fading assumption may not be a good assumption for the desired signal since a line-of-sight path is likely to exist in a microcell. Therefore, it is evident that different statistics are needed to characterize the desired user signal and the interfering signals in a micro or picocellular radio systems.

If the probability density function (PDF) of the total interference $I$ is known, then the outage can readily be obtained. The PDF, $f_{l}(\xi)$, can be expressed as an $L$-fold convolution integral. While there is no analytical solution to this integral ia general, several early papers have taken this approach. Another approach is to use the Laplace transform (LT) of the PDF, i.e., the moment generating function (MGF). If the RVs are independent, the MGF of total interference $I$,

$$
\phi_{I}(s)=\int_{0}^{\infty} \exp (-s \xi) f_{l}(\xi) d \xi
$$

is the product of the individual MGFs. While, in principle, inverting this MGF gives the PDF of $I$, closed-form expressions are difficult or impossible under general conditions. This inversion can be circumvented if the desired signal power is a sum of exponential RVs. From (1), it follows that

$$
P_{\text {out }}=\int_{0}^{\infty} F_{0}(q \xi) f_{I}(\xi) d \xi
$$

where $F_{0}(q \xi)$ is the cumulative density function (CI)F) of $p_{0}$. If the desired signal amplitude is Rayleigh fading, then $p_{0}$ is an exponential RV with a CDF of the form $1-\exp (-x)$.'Therefore, by combining (2) and (3) the outage can be expressed using $\phi_{I}(s)$. If the desired signal amplitude is Nakagami faded with an integral fading index $m$, then $p_{0}$ is a sum of $m$ exponential RVs (i.e., a Gamma RV) with a CDF of the form $1-\sum x^{k} \exp (-x)$. If $f(x)$ and $\phi(s)$ form an LT pair, so do $(-x)^{k} f(x)$ and $\phi^{k}(s)$, the $k$ th derivative. Hence, $\phi_{I}^{k}(s), k=0, \ldots, m-1$, yield the outage. By approximating a Rice RV by a Nakagami RV, the above method can be used for the Rice-faded desired signal. 
These are some of the techniques that have appeared in the research literature.

In this paper, we unify the previous results by expressing the outage as a finite-range integral for all the common fading distributions. The MGFs of the desired and interfering signal powers constitute the integrand. Using the standard mathematical and software packages such as Maple and Matlab, it is extremely simple to evaluate (numerically) the integral with high accuracy. Whereas explicit closed-form solutions tend to require much programming effort. Our approach here is partly motivated by this consideration. Moreover, the integral can be approximated by extremely accurate sums requiring knowledge of the MGF at only a small number of points.

\section{STATISTICAL REPRESENTATIONS OF THE FADING CHANNELS}

Given a random variable $X$, the MGF indicates the expected value of the exponential of $X$, i.e., $\phi(s)=\mathrm{E}\left[e^{-2 X}\right]$. Since our unified approach for computing the outage performance only requires the knowledge of MGF of the received signal power, we next identify the MGFs for several commonly used fading channel models.

\section{A. Rician and Rayleigh Fading}

The MGF for a non-centralized chi-squared RV (Rice distribution) is given by [9, pp. 44],

$$
\phi(s)=\frac{1+K}{1+K+s \bar{p}} \exp \left(\frac{-K s \bar{p}}{1+K+s \bar{p}}\right)
$$

where $K$ denotes the Rice parameter, which is the ratio of the power in the line-of-sight and specular components to that in the diffuse component. In a limiting case when the power in the line-of-sight path approaches zero, the channel reverts to the Rayleigh fading channel. Then the corresponding MGF for Rayleigh fading case can be obtained by substituting $K=0$ in (4).

\section{B. Nakagami-m and Nakagami-q (Hoyt) Fading}

The MGF for the Nakagami-m fading channel can be obtained from [2, eq. (44)],

$$
\phi(s)=\left(\frac{m}{m+s \bar{p}}\right)^{m}, \quad m \geq 0.5
$$

where $m$ denotes the fading figure. It is evident that (5) reduces to the Rayleigh fading case when $m=1$.

It can be easily shown that the MGF of the received power for the Nakagami-q fading is,

$$
\phi(s)=\frac{1}{\sqrt{[s \bar{p}(1+b)+1][s \bar{p}(1-b)+1]}}, \quad-1 \leq b \leq 1
$$

where $b=\left[1-q^{2}\right] /\left[1+q^{2}\right]$ and $q(0 \leq q \leq \infty)$ is the fading parameter. In particular, the Nakagami-q distribution reverts to the Rayleigh and the one-sided normal distribution when $b=0$ and $b=1$, respectively.

\section{Lognormal-Rice and Suzuki Fading}

Expressing the received fading envelope as the product of independent Rice and lognormal distributions, and then applying Hermitian integration, we can show

$$
\begin{aligned}
\phi(s)= & \frac{1}{\sqrt{\pi}} \sum_{i=1}^{N} \frac{w_{i}(1+K)}{1+K+s \mu \exp \left(\sqrt{2} \sigma x_{i}\right)} \\
& \times \exp \left(\frac{-K s \mu \exp \left(\sqrt{2} \sigma x_{i}\right)}{1+K+s \mu \exp \left(\sqrt{2} \sigma x_{i}\right)}\right)+R_{H}
\end{aligned}
$$

where $\sigma$ is the logarithmic standard deviation of shadowing, and $\mu$ is the local mean power. The abscissas $x_{i}$ ( $i$ th root of an $N$ th order Hermite polynomial) and weights $w_{i}$ are tabulated in [10] for $N \leq 20$ and $R_{H}$ is a remainder term.

Since Suzuki distribution is a special case of the lognormal Rician distribution, its MGF is readily obtained by setting $K=0$ in (7).

\section{Lognormal-Nakagami-m Fading}

Similar to our derivation of (7), the MGF of the received power in a Nakagami-m fading channel with lognormal shadowing can be expressed as,

$$
\phi(s)=\frac{1}{\sqrt{\pi}} \sum_{i=1}^{N} \frac{w_{i}}{\left[1+s \mu \exp \left(\sqrt{2} \sigma x_{i}\right) / m\right]^{m}}+R_{H}
$$

Let us define

\section{OUTAGE ANALYSIS}

$$
\gamma=\frac{p_{0}}{q}-\left(\sum_{k=1}^{L} p_{k}\right)
$$

and therefore the MGF of $\gamma$ is given by

$$
\phi_{\gamma}(s)=\phi_{0}(s / q) \prod_{k=1}^{L} \phi_{k}(-s)
$$

where $\phi_{k}(s)$ is the MGF of $p_{k}$. It follows that the outage is given by

$$
P_{\text {out }}=\operatorname{Pr}(\gamma<0)
$$

This probability in the form of (11) can be written as [11],

$$
\begin{aligned}
P_{\text {out }} & =\frac{1}{2 \pi} \int_{-\infty}^{\infty} \frac{\phi_{y}(c+j \omega)}{c+j \omega} d \omega \\
& =\frac{1}{\pi} \int_{0}^{\infty} \frac{\operatorname{Real}\left[(c-j \omega)\left(\phi_{\gamma}(c+j \omega)\right)\right]}{c^{2}+\omega^{2}} d \omega
\end{aligned}
$$

where $j^{2}=-1,0<c<a_{\min }=\min \left\{a_{i} \mid 1 \leq i \leq L\right\}$ with $a_{i}$ being the $i$-th pole of $\phi_{y}(s)$ in the left half plane (i.e., $a_{i}>0$ ). Substituting $\omega=c \tan (\theta / 2)$ in (12), we get

$$
P_{\text {out }}=\frac{1}{2 \pi} \int_{0}^{\pi} \tilde{\phi}(\theta) d \theta
$$

where $\tilde{\phi}($.$) is given by,$

$$
\tilde{\phi}(\theta)=\operatorname{Real}\left[(1-j \tan (\theta / 2)) \phi_{y}(c+j c \tan (\theta / 2))\right]
$$

This new form (i.e., (13)) is both easily evaluated and well suited to numerical integration since it only involves finite integration limits and knowledge of the MGF. The simplicity of this result is to be compared with other more complex forms given in the research literature. Further using variable substitution $\theta=\operatorname{acos}(x)$, and then applying the GCQ formula [10, pp. 889] to the resultant integral, we get

$$
P_{\text {out }}=\frac{1}{2 n} \sum_{i=1}^{n} \tilde{\phi}\left[\frac{(2 i-1) \pi}{2 n}\right]+R_{n}
$$


where the remainder term $R_{n}$ vanishes (decays) very rapidly (i.e., (15) is therefore a rapidly converging series).

Although the value of $c$ can be selected anywhere between 0 . and $a_{m i n}$, it is better to choose it such that $\left|\phi_{y}(c+j \omega)\right|$ decays very rapidly as $\omega \rightarrow \infty$. The highest rate of decay is ensured if $s=c$ is a saddle point; i.e., at $s=c, s^{-1} \phi_{y}(s)$ achieves its minimum on the real axis. However, this optimal value of $c$ requires a numerical search. In practice it is sufficient to use $c=a_{\min } / 2$. For the MGFs listed in the previous section, this value can be determined at once. for $R_{n}$,

In Appendix A of [13] we have derived a simpler expression

$$
R_{n}=\frac{\pi^{2}}{6 n^{2}} \tilde{\phi}^{\prime \prime}(\zeta) \text { for some } 0<\zeta<\pi
$$

This new expression is very attractive for numerical evaluation since it only involves a second order derivative of the $M G$. instead of $2 n$-th order derivative of the MGF using the formisia furnished in [10].

\section{COMPUTATIONAL RESULTS AND REMARKS}

In [11] we have verified the accuracy of (15) since the appi ation of (13) for complicated mobile radio scenarios (e.g., unequal interferers and mixed fading distributions) is straightforward. In particular, we have shown that the rule of thumb for the choice of $c$ does not preclude the usage of (15) because $n$ does not grow too large so as to become unmanageable (even with a relatively large deviation from the optimum value), thus eliminates the need for a precise numerical search. Further, a high accuracy can be easily attained with only a reasonable number of MGF samples (i.e., less than 25 samples) over a wide range of fading environments.

In some of the previous studies (e.g., [3]), the authors have suggested approximating a Rice RV by a Nakagami-m RV to examine the outage performance of cellular mobile radio networks subject to Rician fading with nonidentical CCI statistics. This was done to circumvent the difficulty in performing the L-fold convolution integral to get $f_{I}(\xi)$. Hence, in Figs. 1 and 2 we investigate whether this is a valid (realistic) approximation. In Fig. 1, we compare the outage performance of a cellular network where both the wanted signal and the four interfering signals are Rician-faded with its corresponding Nakagami-m approximation fading model. The fading severity index $m$ (also known as fading figure) is obtained using the relationship $m=(K+1)^{2} /(2 K+1)$. The interference signal statistics are given by $\vec{p}_{k}=[1.1,0.6,0.9,2.2]$ and $K_{k}=[2.5,1.3,3.2,4.7]$. It is apparent from Fig. 1 that the Nakagami ${ }^{1}$ model for the desired signal yields misleadingly optimistic results. This observation may be attributed to the fact that the tails of the Rician

1. It is noted that the real importance of the Nakagami-m fading model lies in the fact that it can often be used to fit experimental data and offers features of analytical convenience in comparison to the Rician distribution. However, the goodness-of-fit tests used by ionospheric physicists to match measured scintillation data to a Nakagami-m distribution do not give special weighting to the deep-fading tail of the distribution. As a result, we sometimes have better fit near the median of the distribution than in the tail region, although the tail behavior is of greater significance to communication systems performance analysis. and its Nakagami-m approximation distributions do not fit very closely. The difference in the behavior below the $1 \%$ poirt of the cumulative distribution function (CDF) of the power for these two distributions (see Fig. 2) may have led to a large difference in the required $S I R / q$ to achieve a prescribed value of $P_{\text {out }}$. Comparison between these CDF curves also suggests that some basic results on Nakagami-m approximation model have been overlooked by some researchers previously.

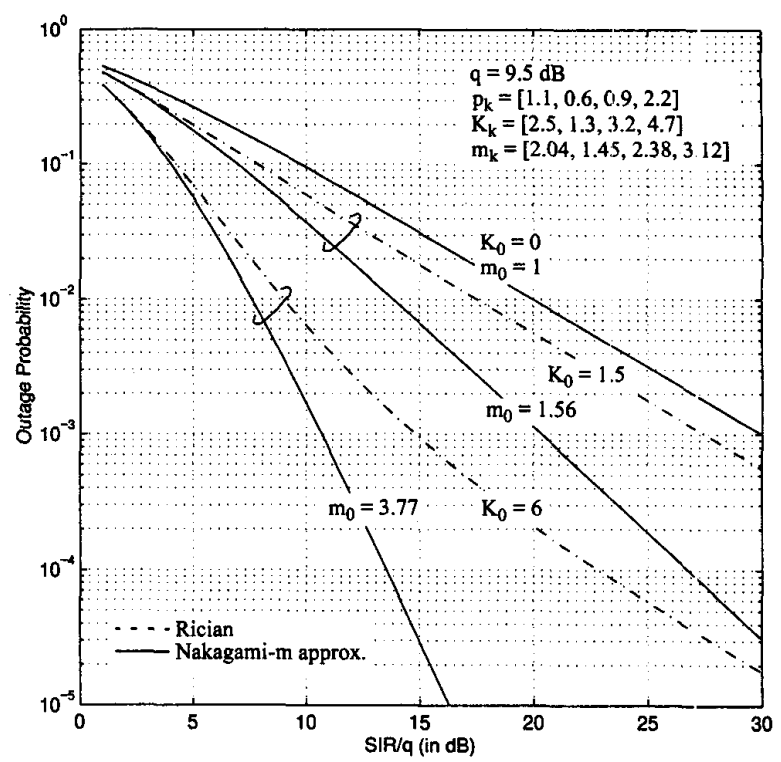

Fig 1. Comparison of the outage probability between the Rician and its Nakagami-m approximation model.

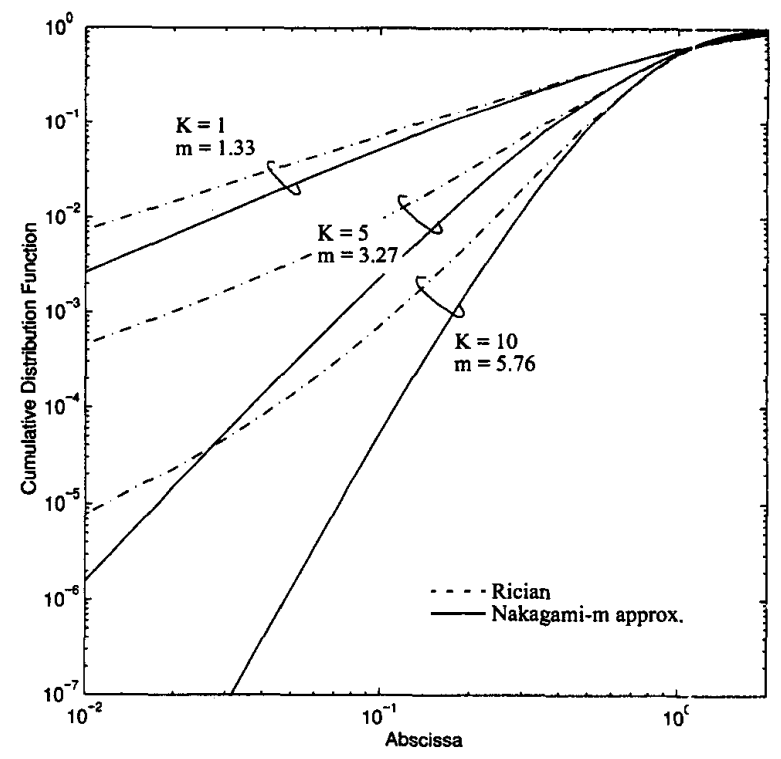

Fig 2. Comparison between the CDFs of the normalized power for the Rician distribution and their corresponding Nakagami-m RVs.

Diesta and Linnartz [4] also obtained a similar result by studying the behavior of the Laplace transform expressions for small arguments (i.e., corresponding to large $S I R$ ) by expanding 
into MacLaurin series. Further, they suggested that Nakagami PDF with $m>1$ does not model deep fades, so it predicts much better performance than Rician fading. Of course the Nakagami-m and Rician models are identical when $K_{0}=0$. From Fig. 1 , we also find that the discrepancy between the actual and approximation model gets larger as $K_{0}$ decreases, for a given outage value. For instance, the difference in the required $S I R / q$ to achieve $P_{\text {out }}=10^{-3}$ is $7.2 \mathrm{~dB}$ and $4.1 \mathrm{~dB}$ when $K_{0}=1.5$ and $K_{0}=6$, respectively. But the Nakagami-m model predicts the exact performance that of Rician distribution when $K=0$. We conclude that approximating a Rician RV of the desired signal by a Nakagami-m is only accurate for its extreme limiting cases -Rayleigh fading $(K=0)$ and nonfading $(K=\infty)$. The discrepancy becomes larger as $S I R / q$ increases. The approximation is inaccurate even at moderate $S I R / q$ values. This is particularly interesting because our unified expression handles the Rician-faded desired signal as well as the mixed-fading situation very well.

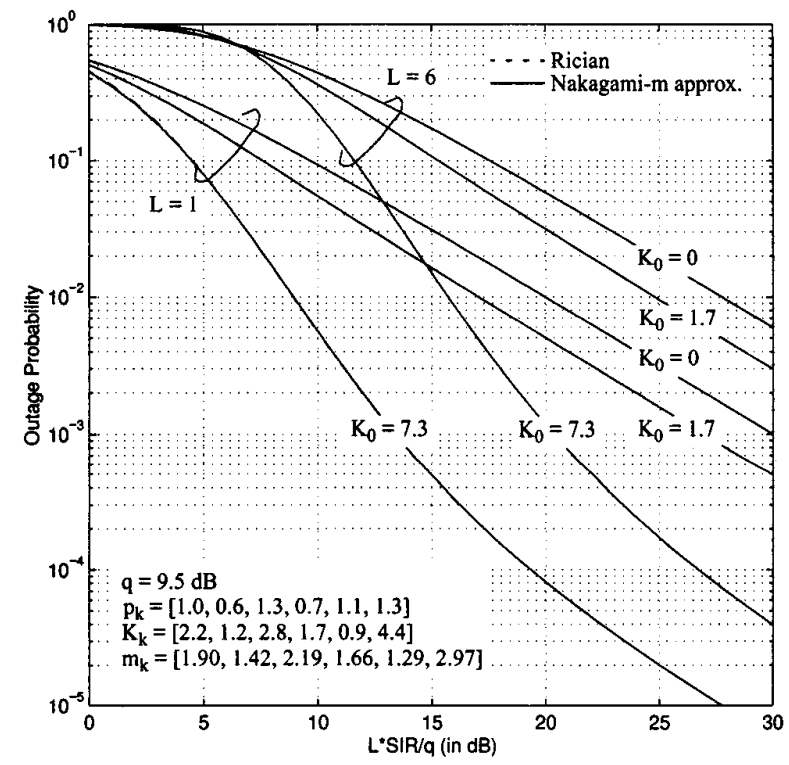

Fig 3. Comparison of the outage between the Rician and its equivalent Nakagami-m approximation model of the interfering signals (the desired signal is modelled as Rician-faded for both cases) for different $K_{0}$ as well as number of interferers $L$.

In Fig. 3, we confirm that one may accurately model the Rician-faded interfering signal by a Nakagami-m signal. Different from Fig. 1, we only model the unwanted Rician-faded signals using its equivalent Nakagami-m approximation and the desired signal is still modelled as Rician-faded (i.e., a mixed Rician/Nakagami fading case). Specifically, we show that the discrepancy observed in our Fig. 1 is attributed to the inaccurate modelling of the desired signal rather than the interfering signal statistics. The curves obtained by modelling the interferers as Nakagami-faded signals yields virtually identical results with that evaluated directly as Rician-faded interfering signals (we cannot distinguish their difference from Fig. 3 because the error due to the approximation is negligible). Fig. 3 also illustrates the effect of increasing the number of interferers on the outage per- formance. Note that the abscissa is the average single interferer power. When $L=1$ the interference statistics $\bar{p}_{1}=1.0$ and $K_{1}=2.2$, which also correspond to the first entry of vectors $\bar{p}_{k}$ and $K_{k}$, respectively. The penalty in the required $S I R / q$ to achieve a prescribed outage gets larger with the $\mathrm{CCI}$ (i.e., higher number of interferers), as anticipated. As well, when the Rice factor of the wanted signal increases, a desired outage is achieved at smaller $S I R / q$ because severity of the signal fading decreases.

In Fig. 4, we further substantiate the trends as well as the conclusions drawn from Fig. 3. In particular, we show that the outage performance is not too sensitive to the variations in the fading severity index of the interfering users. For simplicity, let us assume that all fading statistics of the interfering signals are identical. We see that the outage probability does not vary too much despite a considerable perturbation in the Rice factor of the interfering signals if $K_{0}$ is small or if $L$ is large. This observation in turn explains why there is negligible amount of error of the outage performance by modelling a Rician RV by a Nakagami-m RV for the interferer signals. However, note that the outage probability becomes more susceptible to the inaccuracies in modelling the interfering signals as $K_{0}$ increases when there are only a few number of interferers (i.e., single interferer case) since the spread between the curve gets larger.

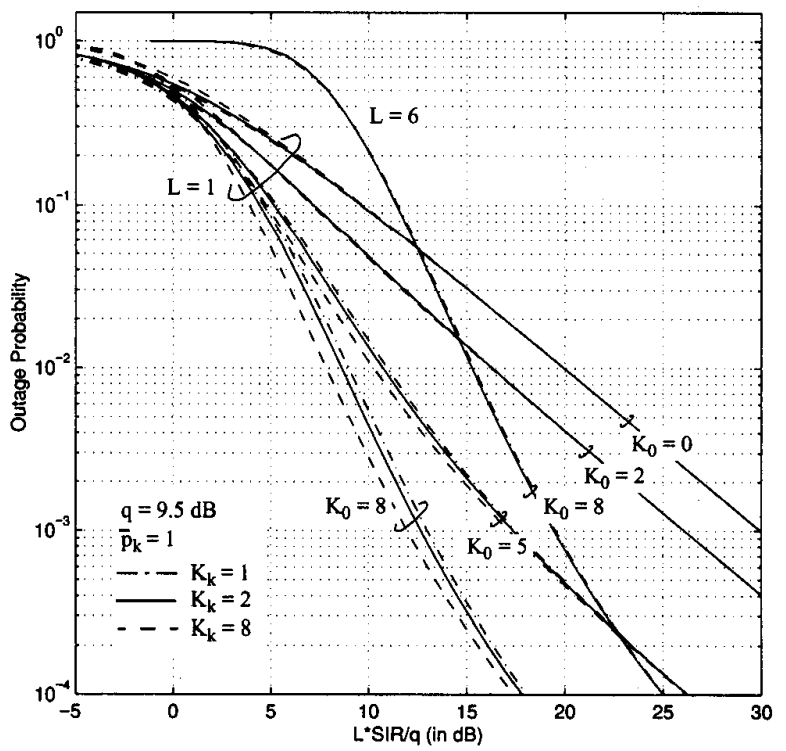

Fig 4. Outage probability versus average signal power to a single interferer power ratio in a Rician (desired)/Rician (interferer) fading channel.

It can also be seen from Fig. 4 that as $K_{k}(k=1, \ldots, L)$ decreases, the outage probability gets larger for a given $S I R / q$ in the moderate range (say $5 \mathrm{~dB}$ to $25 \mathrm{~dB}$ ) if $K_{0}>0$. This can be explained by realizing that for a given $S I R / q, K_{0}$ and $\bar{p}_{k}, \bar{p}_{0}$ must be increased if $K_{k}$ decreases to keep $L \times S I R / q$ the same because

$$
S I R=\frac{\bar{p}_{0}}{\sum_{k} \bar{p}_{k}}=\frac{\left(K_{0}+1\right) \bar{p}_{0(\text { diffused })}}{\sum_{k}\left(K_{k}+1\right) \bar{p}_{k(\text { diffused })}}
$$


where $\bar{p}_{i(\text { diffused })}$ denotes the average power in the diffused path. If $K_{0} » 0$, then the outage probability declines very rapidly even with a slight increase in $\bar{p}_{0}$ (i.e., 'waterfall curve') in the range of interest, which explains the above phenomena. At the other extreme (i.e., when the desired signal is Rayleigh-faded), the outage probability gets larger as $K_{k}$ increases. If we change the abscissa to $\bar{p}_{0}$, then we will find that the outage will always be higher for a larger $K_{k}$, as anticipated.

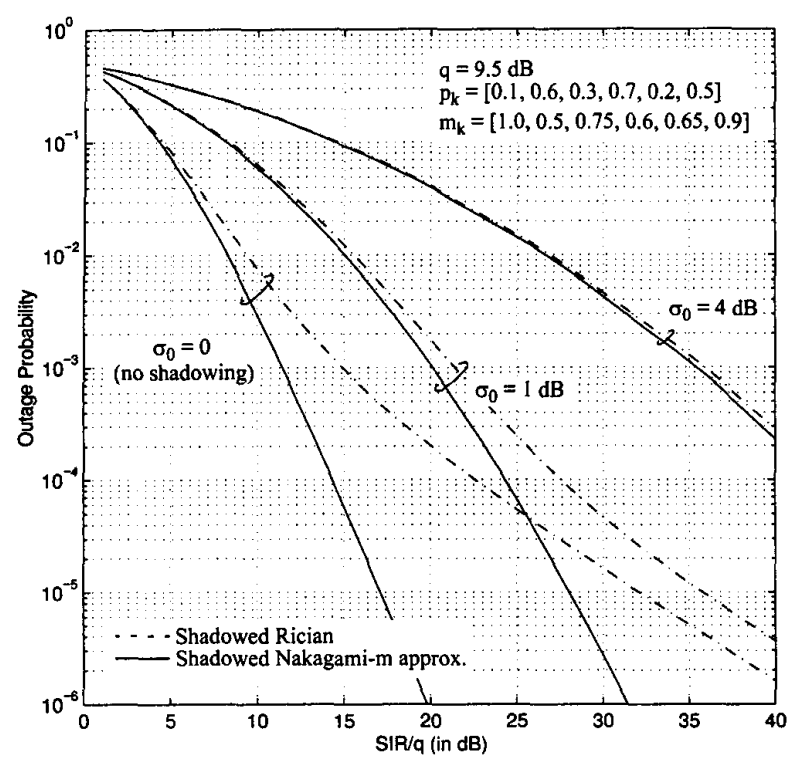

Fig 5. Comparison of the outage between the shadowed Rician-faded desired signal case $\left(K_{0}=6.1\right)$ and its equivalent shadowed Nakagami-m approximation model for different shadowing spreads $\sigma_{0}$ of the desired user signal.

In Fig. 5, we investigate the accuracy of modelling the shadowed Rician-faded desired signal PDF by its corresponding shadowed-Nakagami approximation. Here we assume that all the six interfering signals envelope follow the Nakagami distribution. The Nakagami distribution with fading figure $m<1$ models a fading channel condition that is more severe than the Rayleigh distribution (which cannot be represented using a Rician distribution). The Rice factor of the desired signal is assumed to be $K_{0}=6.1$. Hence, the fading severity parameter of the Nakagami approximation model is $m_{0}=3.819$. From Fig. 4 , we see that the outage probabilities for the shadowed Nakagami-m channel and the shadowed Rician channel have good agreements for small $S I R / q$; otherwise the deviation can be substantial, in particular when the shadowing is not severe. The shadowing has the net effect of shifting the abscissa (i.e., $S I R / q$ ) when the departure begins to the right. Our results reveal that this approximation is plausible only in the limiting case (i.e., exact for the Suzuki fading environment) or if the desired signal experience heavy shadowing (i.e., large $\sigma_{0}$ ). Therefore the approximation performed in [8] is inadequate and cannot be justified for a general fading environment. However, the intuitive claim by the authors that the accuracy of approximating the shadowed Rician-faded desired signal by a shadowed-Nakagami improves when a strong specular path is available is correct.

\section{CONCLUSIONS}

In the research literature, much effort has been expended to find closed-form expressions for outage in mobile radio systems. This ad hoc development has led to various formulas. In contrast, we have developed a unified outage expression (a single finite-range integral) for all common fading distributions. This exact outage expression requires the knowledge of the MiJF. We also provide a new closed-form expression (based on GCQ approximation) that offers a convenient method to evaluate the outage. This general method allows for arbitrary fading farameters as well as dissimilar signal strengths, shadowing spre.zds and so on. It is a powerful tool for outage analysis -- not iniposing any restrictions while being easy to program. Moreover, we have shown that, in contrast to common belief, it is not very accurate to approximate the Rician or the shadowed Rician-faded wanted signal statistics by a Nakagami-m or the shadowed Nakagami-m distribution even at moderate $S I R / q$ specifically if the desired signal is not shadowed heavily. Fortunately, the use of this approximation is unnecessary because our solution can handle Rice fading scenarios quite easily.

\section{ACKNOWLEDGMENT}

The authors are very grateful to Prof. Vijay K. Bhargava of University of Victoria for his continuous encouragement ard guidance throughout this work.

\section{REFERENCES}

[1] J. -P. Linnartz, Narrowband Land-Mobile Radio Networks, Artech, 1993.

[2] A. A. Abu-Dayya and N. C. Beaulieu, "Outage Probabilities of Cellular Mobile Radio Systems with Multiple Nakagami Interferers," IEEE Trans. Vehic. Technology, Vol. 40, pp. 757-767, Nov. 1991.

[3] Y. D. Yao and A. U. H. Sheikh, "Investigation into Cochannel Interference in Microcellular Mobile Radio Systems," IEEE Trans. Vehic. Technology, Vol. 41, pp. 114-123, Feb. 1992.

[4] R. F. Diesta and J. -P. Linnartz, "Using Laplace Transforms to Cornpute Performance of Mobile Radio Links," Proc. PIMRC'94, pp. 301-3115, 1994.

[5] Q. T. Zhang, "Outage Probability of Cellular Mobile Radio in the: Presence of Multiple Nakagami Interferers with Arbitrary Paramete:s," IEEE Trans. Vehic. Technology, Vol. 44, pp. 661-667, August 1995.

[6] C. Tellambura and V. K. Bhargava, "Outage Probability Analysis for Cellular Mobile Radio Systems Subject to Nakagami Fading and Sh adowing," IEICE Trans. Communications, pp. 1416-1423, Oct. 1995.

[7] T. T. Tjhung, C. C. Chai and X. Dong, "Outage Probability for Lognormal-Shadowed Rician Channels," IEEE Trans. Vehic. Technology, Vol. 46, pp. 400-407, May 1997.

[8] L. -C. Wang and C. -T. Lea, "Co-channel Interference Analysis fcr Shadowing Rician Channels," IEEE Commun. Letters, Vol. 2, pp. 67-6!), 1998.

[9] J. G. Proakis, Digital Communications, McGraw-Hill, 3rd. Ed., 1995.

[10] M. Abramowitz and I. A. Stegun, Handbook of Mathematical Functions, National Bureau of Standards, Applied Mathematics Series 55, 1964.

[11] C. Tellambura and A. Annamalai, "A Unified Numerical Aporoach for Computing the Outage Probability for Mobile Radio Systems," to appear in the IEEE Commun. Letters.

[12] E. Biglieri, G. Caire, G. Taricco and J. Ventura-Traveset, "Simf le Method for Evaluating Error Probabilities," IEE Electronics Letters, Vol. 32, pp. 191-192, Feb. 1996.

[13] A. Annamalai, C. Tellambura and V. K. Bhargava, "New, Simple and Accurate Methods for Outage Analysis in Cellular Mobile Rad o Systems - A Unified Approach," submitted to the IEEE Trans. Communications. 